\title{
Nosocomial meningitis in intensive care: a 10-year retrospective study and literature review
}

\author{
Sofia R. Valdoleiros ${ }^{1}$, Cristina Torrão ${ }^{2}$, Laura S. Freitas ${ }^{3}$, Diana Mano ${ }^{2}$, Celina Gonçalves ${ }^{2}$, Carla Teixeira ${ }^{2,4,5}$ \\ ${ }^{1}$ Department of Infectious Diseases, Centro Hospitalar Universitário de São João, Porto; ${ }^{2}$ Department of Intensive Care, Centro Hospitalar Universitário do Porto, \\ Porto; ${ }^{3}$ Department of Emergency, Centro Hospitalar de Entre o Douro e Vouga, Santa Maria da Feira; Instituto de Ciências Biomédicas Abel Salazar, Universidade \\ do Porto, Porto; ${ }^{5}$ CriticalMed Cintesis, Centro de Investigação em Tecnologias e Serviços de Saúde, Faculdade de Medicina da Universidade do Porto, Porto, \\ Portugal
}

Background: Nosocomial meningitis is a medical emergency that requires early diagnosis, prompt initiation of therapy, and frequent admission to the intensive care unit (ICU).

Methods: A retrospective study was conducted in adult patients diagnosed with nosocomial meningitis who required admission to the ICU between April 2010 and March 2020. Meningitis/ventriculitis and intracranial infection were defined according to Centers for Disease Control and Prevention guidelines.

Results: An incidence of $0.75 \%$ of nosocomial meningitis was observed among 70 patients. The mean patient age was 59 years and $34 \%$ were $\geq 65$ years. Twenty-two percent of patients were in an immunocompromised state. A clear predisposing factor for nosocomial meningitis (traumatic brain injury, basal skull fracture, brain hemorrhage, central nervous system [CNS] invasive procedure or device) was present in 93\% of patients. Fever was the most frequent clinical feature. A microbiological agent was identified in $30 \%$ of cases, of which $27 \%$ were bacteria, with a predominance of Gram-negative over Gram-positive. Complications developed in 47\% of cases, 24\% of patients were discharged with a Glasgow coma scale $<14$, and 37\% died. There were no clear clinical predictors of complications. Advanced age ( $\geq 65$ years old) and the presence of complications were associated with higher hospital mortality.

Conclusions: Nosocomial meningitis in critical care has a low incidence rate but high mortality and morbidity. In critical care patients with CNS-related risk factors, a high level of suspicion for meningitis is warranted, but diagnosis can be hindered by several confounding factors.

Key Words: bacterial meningitis; central nervous system infections; critical care; critical illness; healthcare-associated meningitis; hospital infection

\section{INTRODUCTION}

Central nervous system (CNS) infections are medical emergencies that require an early diagnosis, prompt initiation of therapy, and frequent admission to an intensive care unit (ICU) [1]. Nosocomial meningitis causes $40 \%$ of cases of bacterial meningitis in industrialized countries [2] and is associated with high mortality and morbidity [1]. Risk factors include invasive procedures (such as craniotomy, internal ventricular catheters, external ventricular drains (EVD),

\section{Original Article}

Received: August 31, 2021

Revised: October 23, 2021

Accepted: November 8, 2021

\section{Corresponding author}

Sofia R. Valdoleiros

Department of Infectious Diseases, Centro Hospitalar Universitário de São João, Alameda Prof. Hernâni Monteiro, 4200-319 Porto, Portugal Tel: +351-222-077-500

E-mail:sofia.valdoleiros@chsj.minsaude.pt
Copyright () 2022 The Korean Society of Critical Care Medicine

This is an Open Access article distributed under the terms of Creative Attributions Non-Commercial License (https:// creativecommons.org/li-censes/by-nc/4.0/) which permits unrestricted noncommercial use, distribution, and reproduction in any medium, provided the original work is properly cited. 
external lumbar catheters, and lumbar puncture) [3-5], moderate or severe head trauma [6-8], subarachnoid hemorrhage $[9,10]$, and, in rare cases, metastatic infection in patients with bacteremia [4]. Tumor neurosurgery, severe traumatic brain injury (TBI), and subarachnoid hemorrhage carry the highest risk for postoperative meningitis [2]. Possible clinical characteristics of nosocomial meningitis include fever, headache onset, nausea, change in mental status, evidence of meningeal irritation, and seizures [11]. Nevertheless, diagnosing nosocomial meningitis is challenging and particularly problematic in the ICU.

Patients in ICUs are often sedated or unresponsive due to other conditions or underlying neurologic diseases, which impairs clinical assessment. Additionally, fever or increase in acute phase proteins can have several potential foci, infectious or non-infectious, including injury to the thermoregulation center [12]. Infections associated with cerebrospinal fluid (CSF) shunts can cause nonspecific symptoms such as general malaise or low-grade fever [4]; signs of meningeal irritation are present in less than $50 \%$ of patients.[4]

On the other hand, CSF biochemical profiles can be drastically altered by intracerebral hemorrhage, immunosuppression [12], and local inflammatory reactions to blood breakdown products or chemicals after neurosurgical interventions (expected presence of elevated proteinorrachia and pleocytosis [13]). A previous study revealed that the CSF characteristics in post-neurosurgical patients were similar between aseptic meningitis and bacterial meningitis cases, including glucose level [13]. In patients with EVD, severe disturbances in the CSF limit the value of CSF analysis for prediction or diagnosis of EVD-related meningitis [14]. Frequent prior or concomitant use of antimicrobials in the ICU further complicates interpretation of the CSF tests; although cell counts in CSF are useful, they have low sensitivity and specificity in some clinical subgroups [14].

CSF culture is considered the gold standard for diagnosing bacterial meningitis. However, its clinical use in ICU populations is limited by frequent prior antibiotic administration, which decreases culture yield. Infections that require immediate therapeutic attention must be differentiated from contamination or device colonization [12]. In up to $70 \%$ of meningitis cases that develop after intraventricular hemorrhage, meningitis is aseptic [13].

To assess the impact of nosocomial meningitis in intensive care patients, a retrospective study was conducted. Aims included estimating disease incidence and identifying risk fac-

\section{KEYMESSAGES}

- Nosocomial meningitis in critical care has a low incidence rate but high mortality and morbidity.

- In this retrospective study of nosocomial meningitis, fever was the most frequent clinical sign.

- Advanced age and complications were associated with higher mortality.

tors for worse outcomes.

\section{MATERIALS AND METHODS}

This study was approved by the Ethics Committee and by the Administrative Council of CHUPorto (No. 2019.317). Individual informed consent was waived.

In this retrospective, single-center study, we reviewed all adult patients (over 18 years of age) diagnosed with nosocomial meningitis who were admitted to the ICU at University Hospital Center of Porto-CHUPorto between April 2010 and March 2020. CHUPorto is a central and university teaching hospital (in association with the University of Porto) with over 800 beds, 24 of which are ICU beds. The hospital admits 35,000 patients per year. As a tertiary center, it serves a population of over 3,000,000 people.

Meningitis, ventriculitis, and intracranial infection were defined according to the guidelines of Centers for Disease Control and Prevention (CDC) (Supplementary Table 1) [15]. Infection acquisition was considered nosocomial when it occurred at least 48 hours after admission. Altered mental status was defined as not being alert on "alert, verbal, pain, unresponsive (AVPU)" score or by a Glasgow coma scale (GCS) score $<14$ points.

Statistical analyses were performed with IBM SPSS ver. 26 (IBM Corp., Armonk, NY, USA). The Kolmogorov-Smirnov test of normality was used to assess whether continuous variables had a normal or non-normal distribution; Student t-test and the Mann-Whitney U-test were performed accordingly. To compare categorical variables, Fisher's exact test and chisquare test were applied. The presence of complications, nonalert state at discharge ("AVPU" score), and mortality were established outcomes. Univariate and multivariate analyses were performed to assess the associations of significant variables with hospital and 30-day mortalities. Statistical significance was accepted at $\mathrm{P}<0.05$. 


\section{RESULTS}

\section{Descriptive Analysis}

Between April 2010 and March 2020, 10,664 adult patients were admitted to the ICU; of these, 80 had a diagnosis of nosocomial meningitis $(0.75 \%)$. Ten patients were excluded from the study due to insufficient data or because they did not fulfill the diagnostic criteria for CNS infection (Figure 1).

Study population characteristics are presented in Table 1. The population had a mean age of $59 \pm 16$ years (range, 18-88), with a large proportion (34.3\%) of elderly patients ( $\geq 65$ years old). The population was predominantly male (62.9\%). Predisposing underlying conditions included alcohol abuse $(n=13$, $18.6 \%)$, diabetes mellitus ( $n=9,12.9 \%)$, immunosuppressive therapy $(n=3,4.3 \%)$, primary immunodeficiency $(n=1,1.4 \%)$, and drug abuse $(\mathrm{n}=1,1.4 \%)$.

Of the 70 included patients, 69 met the diagnostic criteria for meningitis/ventriculitis and one for intracranial infection with meningitis (Figure 1). Sixty-five patients (93\%) had a clear CNS-related predisposing factor for nosocomial meningitis (TBI, basal skull fracture, brain hemorrhage, or CNS invasive procedure); of the five remaining patients $(7.1 \%)$, two had bac-

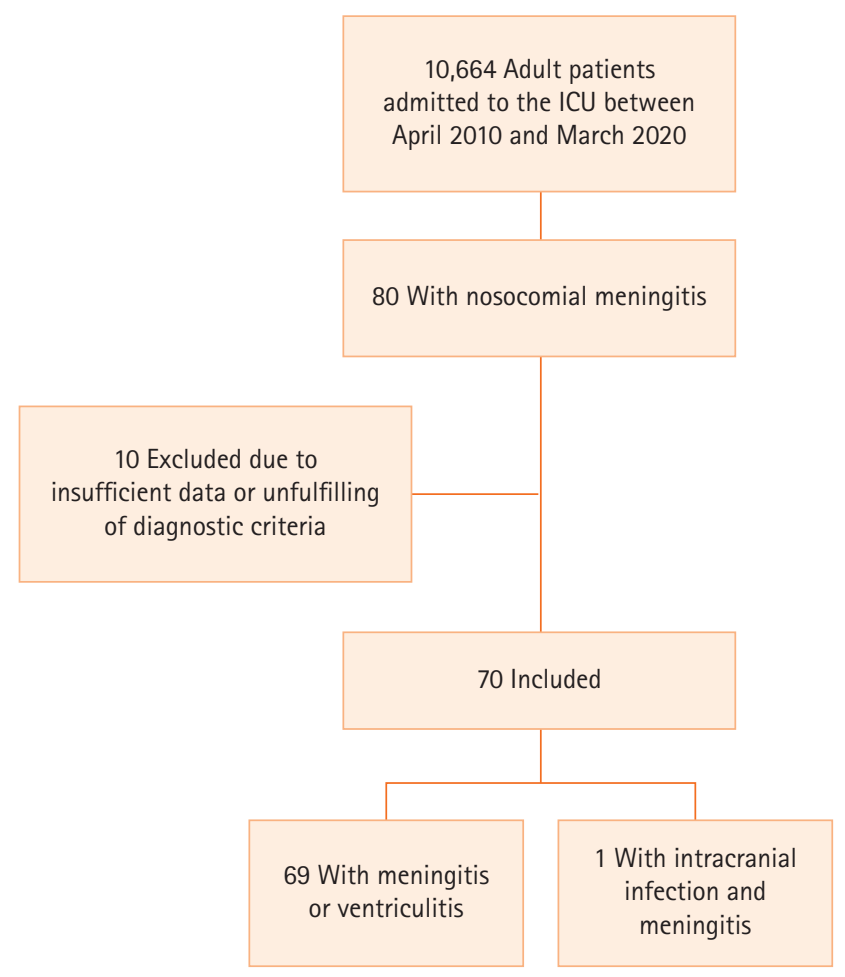

Figure 1. Flowchart of patients included in the study. ICU: intensive care unit. teremia, one had endocarditis, and one had a dental abscess. Invasive $\mathrm{CNS}$ procedure was the most common risk factor for nosocomial meningitis ( $\mathrm{n}=62,88.6 \%)$.

Clinical and laboratory characteristics of CNS infection are depicted in Table 2. Fever was the most common clinical feature, occurring in $90 \%$ of patients. Seizures and new focal deficits (hemiparesis) were observed in 11 (15.7\%) and five (7.1\%) patients, respectively. Among non-sedated patients $(n=38)$, altered mental status (defined as not alert on AVPU score or GCS $<14$ points) was the second most frequent symptom $(n=27)$.

Lumbar puncture was performed in most patients. CSF analysis disclosed a mean glucose level of $0.69 \pm 0.35 \mathrm{~g} / \mathrm{L}$, median protein of $1.15 \mathrm{~g} / \mathrm{L}$ (interquartile range [IQR], 0.65-1.84), and median white cell count of $359 / \mu \mathrm{L}$ (IQR, 132-1,634) (Table 2).

Table 1. Patients' characteristics

\begin{tabular}{|c|c|}
\hline Demographics & Value \\
\hline Age (yr) & $59 \pm 16$ \\
\hline Age $\geq 65 \mathrm{yr}$ & $24(34.3)$ \\
\hline Male & $44(62.9)$ \\
\hline Functional status: independent & 64 (91.4) \\
\hline \multicolumn{2}{|l|}{ Underlying disease } \\
\hline Immunosuppressive therapy & $3(4.3)$ \\
\hline Primary immunodeficiency & $1(1.4)$ \\
\hline Diabetes mellitus & $9(12.9)$ \\
\hline Type 2, insulin-treated & $2(2.9)$ \\
\hline Type 2, non-insulin-treated & $7(10.0)$ \\
\hline Alcohol abuse & $13(18.6)$ \\
\hline Drug abuse & $1(1.4)$ \\
\hline \multicolumn{2}{|l|}{ Predisposing condition } \\
\hline $\mathrm{TBI}$ & $24(34.3)$ \\
\hline Basal skull fracture & $7(10.0)$ \\
\hline CNS invasive procedure & $62(88.6)$ \\
\hline Craniotomy & $31(44.3)$ \\
\hline Craniectomy & $17(24.3)$ \\
\hline Cranioplasty & $1(1.4)$ \\
\hline Spinal surgery & $4(5.7)$ \\
\hline CNS invasive device & $45(64.3)$ \\
\hline EVD & 23 (32.8) \\
\hline ICP-monitoring catheter & $32(45.7)$ \\
\hline Lumbar drain & $4(5.7)$ \\
\hline Distant focus of infection & $2(2.9)$ \\
\hline Odontogenic abscess & $1(1.4)$ \\
\hline Endocarditis & $1(1.4)$ \\
\hline
\end{tabular}

Values are presented as mean \pm standard deviation or number (\%). TBI: traumatic brain injury; CNS: central nervous system; EVD: external ventricular drain; ICP: intracranial pressure. 
Table 2. Clinical and laboratory characteristics of CNS infection

\begin{tabular}{|c|c|}
\hline Variable & Value \\
\hline \multicolumn{2}{|l|}{ Sign and symptom } \\
\hline Fever & $63(90)$ \\
\hline Nausea or vomiting & $4(5.7)$ \\
\hline Meningism & $3(4.3)$ \\
\hline Seizure & $11(15.7)$ \\
\hline Partial seizures & $6(8.6)$ \\
\hline Non-convulsive status epilepticus & $4(5.7)$ \\
\hline Generalized tonic-clonic seizures & $1(1.4)$ \\
\hline Non-sedated patient & $38(54.3)$ \\
\hline Headache & $3(4.3)$ \\
\hline Behavior change & $6(8.6)$ \\
\hline New focal deficits & $5(7.1)$ \\
\hline \multicolumn{2}{|l|}{ "AVPU" at diagnosis } \\
\hline Alert & $11(15.7)$ \\
\hline Responsive to verbal stimuli & $4(5.7)$ \\
\hline Responsive to pain & $15(21.4)$ \\
\hline Unresponsive & $8(11.4)$ \\
\hline \multicolumn{2}{|l|}{ Glasgow coma score at diagnosis } \\
\hline $3-8$ points & $13(18.6)$ \\
\hline 9-13 points & $15(21.4)$ \\
\hline 14-15 points & $8(11.4)$ \\
\hline \multicolumn{2}{|l|}{ Timing of symptoms and diagnosis } \\
\hline Time between hospital admission and diagnosis & $10.9 \pm 8.6(1-48)$ \\
\hline Time until readmission & $9.2 \pm 4.6(2-13)$ \\
\hline Time between any predisposing factor and signs/symptoms & $6.7 \pm 5.8(0-25)$ \\
\hline Time between TBI and signs/symptoms & $6.6 \pm 6.5(2-34)$ \\
\hline Time between basal skull fracture and signs/symptoms & $6.2 \pm 4.2(2-12)$ \\
\hline Time between CNS invasive procedure and signs/symptoms & $6.5 \pm 5.6(0-25)$ \\
\hline Time between CNS invasive device and signs/symptoms & $5.5 \pm 6.0(0-41)$ \\
\hline Time between symptoms and diagnosis & $2.0 \pm 2.1(0-10)$ \\
\hline Time between any predisposing factor and diagnosis & $9.0 \pm 7.5(2-43)$ \\
\hline Time between TBI and diagnosis & $8.9 \pm 6.5(2-37)$ \\
\hline Time between basal skull fracture and diagnosis & $7.3 \pm 4.2(2-13)$ \\
\hline Time between CNS invasive procedure and diagnosis & $8.7 \pm 6.1(2-29)$ \\
\hline Time between CNS invasive device and diagnosis & $7.4 \pm 6.2(0-43)$ \\
\hline \multicolumn{2}{|l|}{ Blood panel } \\
\hline White cell count $\left(/ \mathrm{mm}^{3}\right)$ & $11,690 \pm 5,516(980-27,660)$ \\
\hline Neutrophils $\left(/ \mathrm{mm}^{3}\right)$ & $9,226 \pm 4,572(630-22,740)$ \\
\hline C-reactive protein (mg/L) & $127 \pm 93(13-493)$ \\
\hline Procalcitonin (ng/ml) & $0.24(0.17-0.78)$ \\
\hline Albumin (g/dl) & $2.9 \pm 0.46(1.65-4.00)$ \\
\hline \multicolumn{2}{|l|}{ CSF analysis } \\
\hline Glucose $(\mathrm{g} / \mathrm{L})$ & $0.69 \pm 0.35(0.01-1.45)$ \\
\hline Protein (g/L) & $1.15(0.65-1.84)$ \\
\hline Red cell $(/ \mu l)$ & $24,300(2,350-89,500)$ \\
\hline White cell $(/ \mu l)$ & $359(132-1,634)$ \\
\hline Polymorphonuclear cell $(/ \mu l)$ & $255(69-1,021)$ \\
\hline Mononuclear cell $(/ \mu l)$ & $106(31-294)$ \\
\hline
\end{tabular}

Values are presented as number (\%), mean \pm standard deviation (range), or median (interquartile range).

CNS: central nervous system; TBI: traumatic brain injury; CSF: cerebrospinal fluid. 
Only two patients had measurable CSF lactate (4.15 and 4.30 $\mathrm{mmol} / \mathrm{L})$. Sixty patients (85.7\%) presented with hypoalbuminemia $(<3.4 \mathrm{~g} / \mathrm{dL})$.

A microbiological agent was identified in 21 patients $(30 \%)$ (Table 3$)$. Only nine patients $(12.9 \%$ ) had a positive CSF culture. Fifty patients $(71.4 \%)$ were treated with antibiotics in the week before diagnosis; of the patients with sterile CSF cultures, 38 (67\%) were treated with antibiotics during the week before diagnosis. Bacteria were identified in 19 cases $(27.1 \%)$, with a predominance of Gram-negative $(n=13,18.6 \%)$ over Gram-positive $(n=8,11.4 \%)$ bacteria. Three infections were polymicrobial, and there was one mixed bacterial and fungal infection. Two infections were strictly fungal, one by Candida albicans and another by Candida parapsilosis. Two strains of Staphylococcus epidermidis were multi-drug resistant, and one strain of Escherichia coli produced extended-spectrum beta-lactamases.

Table 3. Microbiological results

\begin{tabular}{|c|c|}
\hline Microbiologic result & №. $(\%)$ \\
\hline Positive culture & $21(30.0)$ \\
\hline CSF & $9(12.9)$ \\
\hline Blood & $12(17.1)$ \\
\hline Other (CNS biopsy, exudate) & $5(7.1)$ \\
\hline \multicolumn{2}{|l|}{ Infectious agent } \\
\hline Candida albicans & $2(2.9)$ \\
\hline Candida parapsilosis & $1(1.4)$ \\
\hline Citrobacter braakii & $1(1.4)$ \\
\hline Corynebacterium species & $1(1.4)$ \\
\hline Enterobacter aerogenes & $1(1.4)$ \\
\hline Enterobacter cloacae & $2(2.9)$ \\
\hline Enterococcus faecalis & $1(1.4)$ \\
\hline Enterococcus faecium & $1(1.4)$ \\
\hline Escherichia coli & $4(5.7)$ \\
\hline Klebsiella pneumoniae & $3(4.3)$ \\
\hline Pseudomonas aeruginosa & $1(1.4)$ \\
\hline Serratia marcescens & $2(2.9)$ \\
\hline Staphylococcus aureus & $2(2.9)$ \\
\hline Staphylococcus epidermidis & $3(4.3)$ \\
\hline \multicolumn{2}{|l|}{ Etiology } \\
\hline Strictly bacterial & $18(25.7)$ \\
\hline Gram-negative bacterial infection & $11(15.7)$ \\
\hline Gram-positive bacterial infection & $6(8.6)$ \\
\hline Both (Gram-positive and Gram-negative) & $1(1.4)$ \\
\hline Fungal & $2(2.9)$ \\
\hline Mixed fungal and bacterial & $1(1.4)$ \\
\hline
\end{tabular}

CSF: cerebrospinal fluid; CNS: central nervous system.
Patients were treated with antimicrobial therapy for an average of $15 \pm 9$ days (range, 1-52); when patients with fewer than 10 days of therapy were excluded (minimum accepted treatment duration), mean antimicrobial therapy was $18 \pm 8$ days (range, 10-52) (Table 4). One patient was treated with intraventricular therapy after having no response to intravenous therapy. Only two patients had their device removed because of infection. Twenty-one patients (30\%) required neurosurgery after diagnosis; however, only five were directly related to CNS infection (drainage of empyema or abscess, subdural space lavage, surgical wound debridement, or closure of CSF fistula) and two underwent decompressive craniectomy. Sixty-four patients (91.4\%) required endotracheal intubation, but only $17(24.3 \%)$ required it after developing CNS infection signs or symptoms.

Patient outcomes are presented in Table 5. Median hospital stay length was 38 days (IQR, 30-46; range, 7-408), and mean ICU stay was $18 \pm 12$ days (range, 1-53). Of the patients who developed nosocomial meningitis during the ICU stay, diagnosis was established, on average, $8 \pm 6$ days after ICU admission (range, $0-43)$. Only five patients $(7.1 \%)$ required readmission to the ICU, of whom two were due to CNS infection; mean time to ICU readmission after discharge was $9 \pm 5$ days (range, 2-13). Thirty-three patients (47.1\%) developed complications; 17

Table 4. Treatment

\begin{tabular}{lc}
\hline Treatment & Value \\
\hline $\begin{array}{l}\text { Empirical antimicrobial therapy } \\
\text { Ceftazidime plus vancomycin }\end{array}$ & $42(60)$ \\
Meropenem plus vancomycin & $22(31.4)$ \\
Route of therapy & \\
$\quad$ Intravenous only & $69(98.6)$ \\
$\quad$ Intravenous and intraventricular & $1(1.4)$ \\
Duration of antibiotic therapy (day) & $18.0 \pm 8.3$ \\
Other therapeutic measure & \\
Corticoid & $13(19.4)$ \\
Intracranial hypertension management & \\
$\quad$ Antiedematous therapy & $8(11.4)$ \\
Barbiturate coma therapy & $2(2.8)$ \\
CSF shunt & $11(15.7)$ \\
Craniectomy & $2(2.9)$ \\
Neurosurgery for infection control & $4(5.7)$ \\
Removal of CNS device & $2(2.9)$ \\
Closure of CSF fistula & $1(1.4)$ \\
\hline
\end{tabular}

Values are presented as number (\%) or mean \pm standard deviation. CSF: cerebrospinal fluid; CNS: central nervous system.

aExlusion of patients treated with $<10$ days of antibiotics. 
Table 5. Outcomes

\begin{tabular}{lc}
\hline Complication & Value \\
\hline Any & $33(47.1)$ \\
Organ failure & \\
$\quad$ Respiratory failure & $37(52.9)$ \\
$\quad$ Shock with vasopressor support & $21(30)$ \\
$\quad$ Acute renal failure & $11(15.7)$ \\
$\quad$ Hematologic dysfunction & $12(17.1)$ \\
$\quad$ Hepatic dysfunction & $8(11.4)$ \\
Intracranial hypertension & $14(20)$ \\
Hydrocephalus & $6(8.6)$ \\
Brain abscess & $2(2.9)$ \\
Cerebral venous sinus thrombosis & $2(2.9)$ \\
Cerebral vasculitis & $1(1.4)$ \\
Amaurosis & $1(1.4)$ \\
Glasgow coma score at discharge & \\
3-8 Points & $4(5.7)$ \\
9-13 Points & $13(18.6)$ \\
14-15 Points & $35(50.0)$ \\
Mortality & \\
Death during hospitalization & \\
Death during ICU & $15(21.4)$ \\
Death within 30 days after admission & $10(14.3)$ \\
Death during and after hospitalization & $15(21.4)$ \\
Rength of stay & $26(37.1)$ \\
Readispital length of stay in days & \\
\hline
\end{tabular}

Values are presented as number (\%), median (interquartile range), or mean \pm standard deviation (range).

ICU: intensive care unit; CNS: central nervous system.

patients $(24.2 \%)$ were discharged with GCS $<14$. Twenty-six patients died (mortality rate, $37.1 \%$ ): 15 during hospital admission (10 during ICU admission) and within the first 30 days after CNS infection diagnosis (in-hospital and 30-day mortality rate, $21.4 \%$ ), and the remaining 11 patients died after hospital discharge.

\section{Comparative Analysis}

Comparative analysis results are shown in Table 6. Regarding complications, we found no association with age, immunocompromised status, clinical features, or laboratory findings. Use of meropenem over ceftazidime, both in association with vancomycin, and therapy with steroids were associated with complications (odds ratio [OR], 3.15; 95\% confidence interval $[\mathrm{CI}], 1.08-9.22 ; \mathrm{P}=0.033$ and $\mathrm{OR}, 3.54 ; 95 \% \mathrm{CI}, 0.97-12.96$; $\mathrm{P}=0.048$, respectively). Antiedematous therapy was only used for patients with complications.

The presence of complications, GCS $<8$ at diagnosis, and antiedematous therapy were not associated with non-alert state at discharge. The two patients who presented with focal deficits at diagnosis and survived were discharged in a non-alert state $(\mathrm{P}=0.08)$. Male patients were more likely to be discharged in a non-alert state (OR, 7.60; 95\% CI, 0.89-64.62; $\mathrm{P}=0.04)$.

Age had no significant impact on patient outcomes, but subgroup analysis of elderly patients ( $\geq 65$ years old) revealed higher hospital mortality and 30-day mortality (OR, 8.89; 95\% CI, 2.42-32.68; $\mathrm{P}=0.001$ ). Partially dependent patients had higher hospital and 30-day mortality (OR, 9.64; 95\% CI, 1.57-59.32; $\mathrm{P}=0.017$ ). An immunocompromised state was not associated with higher mortality (OR, 1.03; 95\% CI, 0.31-3.46; $\mathrm{P}=1.000$ ). Patients who developed complications had higher hospital and 30-day mortality without statistical significance on univariate analysis (OR, 2.78; 95\% CI, 0.83-9.24; $\mathrm{P}=0.087$ ).

In multivariate analysis (Table 7), the associations of complications with hospital and 30-day mortalities were statistically significant (OR, 5.04; 95\% CI, 1.01-25.19; $\mathrm{P}=0.049$ ). Multivariate analysis of the relationships of age $\geq 65$ years with hospital and 30-day mortality also presented statistical significance (OR, 10.08; 95\% CI, 2.03-50.03; $\mathrm{P}=0.005)$.

\section{DISCUSSION}

Accurate estimates of nosocomial meningitis incidence are hampered by variations in diagnosis, infection definition, institutional infection control, and device manufacturers [1]. Reported incidences vary widely, with overall incidence ranging from $<1 \%-7 \%$, and with postoperative meningitis developing in $0.34 \%-25 \%$ of cases $[3,16,17]$. We observed an overall incidence within this range, of $0.75 \%$, in critical care patients. To the best of our knowledge, this is the first study of exclusively nosocomial meningitis in a general ICU population that includes both postoperative and non-postoperative patients; thus, we cannot directly compare this incidence with those of other studies.

Evidence of association between demographic characteristics and outcomes in nosocomial meningitis is limited. While Dizbay et al. [18] found higher mortality in younger patients, Srihawan et al. [19] reported that age $\geq 45$ years was associated with higher risk of adverse clinical outcomes, and Lu et al. [20] 
Table 6. Univariate analysis

\begin{tabular}{|c|c|c|c|c|c|c|}
\hline \multirow{2}{*}{ Variable } & \multicolumn{2}{|c|}{ Any complication } & \multicolumn{2}{|c|}{ Non-alert at discharge ${ }^{a}$} & \multicolumn{2}{|c|}{$\begin{array}{c}\text { 30-Day mortality and hospital } \\
\text { mortality }\end{array}$} \\
\hline & OR $(95 \% \mathrm{Cl})$ & P-value & OR $(95 \% \mathrm{Cl})$ & P-value & $\mathrm{OR}(95 \% \mathrm{Cl})$ & P-value \\
\hline \multicolumn{7}{|l|}{ Demographics and underlying disease } \\
\hline Age $\geq 65 \mathrm{yr}$ & $0.71(0.26-1.93)$ & 0.51 & $1.30(0.28-5.73)$ & 0.71 & $8.89(2.42-32.68)$ & 0.001 \\
\hline Male & $1.06(0.40-2.82)$ & 0.90 & $7.60(0.89-64.62)$ & 0.04 & $0.86(0.27-2.76)$ & 1 \\
\hline Previous partially dependent & $0.53(0.09-3.12)$ & 0.68 & $0.80(0.69-0.91)$ & 1 & $9.64(1.57-59.32)$ & 0.02 \\
\hline Immunocompromised & $0.80(0.29-2.19)$ & 0.67 & $1.98(0.51-7.68)$ & 0.47 & $1.03(0.31-3.46)$ & 1 \\
\hline \multicolumn{7}{|l|}{ Clinical feature } \\
\hline Fever at diagnosis & $0.16(0.01-1.1)$ & 0.06 & $1.28(1.11-1.48)$ & 0.57 & $0.65(0.11-3.74)$ & 0.64 \\
\hline GCS at diagnosis $<8$ points ${ }^{b}$ & $0.68(0.23-2.00)$ & 0.48 & $4.09(0.83-20.14)$ & 0.71 & $0.94(0.27-3.22)$ & 1 \\
\hline New focal neurological deficit at diagnosis ${ }^{b}$ & $5.67(0.56-57.23)$ & 0.16 & - & 0.08 & $3.94(0.55-28.11)$ & 0.3 \\
\hline Complication (any) & NA & NA & $0.45(0.11-1.92)$ & 0.33 & $2.78(0.83-9.24)$ & 0.09 \\
\hline Hospital stay (mean) & $2(-37$ to 13$)$ & 0.34 & 35 (-94 to 61$)$ & 0.65 & $30.4(12.70-48.05)$ & 0.05 \\
\hline \multicolumn{7}{|l|}{ Laboratory findings } \\
\hline C-reactive protein $\geq 100 \mathrm{mg} / \mathrm{L}$ & $1.29(0.50-3.31)$ & 0.60 & $1.00(0.26-3.77)$ & 1 & $0.95(0.30-2.99)$ & 0.93 \\
\hline CSF white cell $\geq 100$ per $\mu \mathrm{l}$ & $1.10(0.33-3.61)$ & 0.88 & $3.12(0.36-27.50)$ & 0.42 & $0.79(0.18-3.40)$ & 0.71 \\
\hline CSF glucose level $<0.30 \mathrm{~g} / \mathrm{L}$ & $0.99(0.24-4.09)$ & 1 & $3.56(0.66-19.11)$ & 0.15 & $1.31(0.24-7.29)$ & 0.67 \\
\hline CSF protein level $\geq 1 \mathrm{~g} / \mathrm{L}$ & $1.52(0.55-4.17)$ & 0.42 & $0.51(0.13-1.96)$ & 0.49 & $1.42(0.38-5.31)$ & 0.75 \\
\hline Concomitant bacteremia & $1.15(0.33-3.98)$ & 0.83 & $0.63(0.07-5.88)$ & 1 & $3.43(0.90-13.02)$ & 0.11 \\
\hline Negative CSF culture & $0.56(0.20-1.58)$ & 0.27 & $0.78(0.18-3.52)$ & 0.71 & $0.27(0.08-0.89)$ & 0.05 \\
\hline \multicolumn{7}{|l|}{ Treatment } \\
\hline Meropenem plus vancomycin ${ }^{c}$ & $3.15(1.08-9.22)$ & 0.03 & $0.96(0.21-4.30)$ & 1 & $2.25(0.63-8.06)$ & 0.31 \\
\hline Antibiotic treatment $>14$ days $^{d}$ & $2.84(0.91-8.86)$ & 0.07 & $1.48(0.29-7.54)$ & 0.70 & $1.05(0.19-5.74)$ & 1 \\
\hline Steroid therapy & $3.54(0.97-12.96)$ & 0.05 & $4.34(0.93-20.30)$ & 0.07 & $2.22(0.56-8.82)$ & 0.26 \\
\hline Antiedematous therapy (including barbiturates) & - & 0.001 & $1.41(0.24-8.16)$ & 0.65 & - & 0.19 \\
\hline CSF shunt & $1.42(0.39-5.18)$ & 0.59 & $0.45(0.05-4.04)$ & 0.67 & $0.79(0.15-4.10)$ & 1 \\
\hline Neurology consult & $0.92(0.34-2.48)$ & 0.87 & $5.25(1.29-21.40)$ & 0.03 & $1.37(0.42-4.44)$ & 0.60 \\
\hline Neurosurgery consult & $1.69(0.45-6.40)$ & 0.44 & $1.58(0.17-14.66)$ & 1 & $0.40(0.10-1.61)$ & 0.23 \\
\hline
\end{tabular}

OR: odds ratio; Cl: confidence interval; GCS: Glasgow coma scale; NA: non-applicable; CSF: cerebrospinal fluid.

${ }^{a}$ Deceased patients excluded; ${ }^{b}$ Sedated patients excluded; Versus ceftazidime plus vancomycin; ${ }^{\text {Ex }}$ Eclusion of patients treated with $<10$ days of antibiotics.

Table 7. Multivariate analysis for 30-day mortality and hospital mortality

\begin{tabular}{lcc}
\hline Variable & OR $(95 \% \mathrm{CI})$ & P-value \\
\hline Age $\geq 65 \mathrm{yr}$ & $10.08(2.03-50.03)$ & 0.01 \\
Male & $1.15(0.25-5.32)$ & 0.86 \\
Previous partially dependent & $4.45(0.42-46.70)$ & 0.21 \\
Complications (any) & $5.04(1.01-25.19)$ & 0.05 \\
Negative CSF culture & $0.53(0.07-1.30)$ & 0.11 \\
\hline
\end{tabular}

OR: odds ratio; $\mathrm{Cl}$ : confidence interval; CSF: cerebrospinal fluid.

reported higher mortality rate in patients $\geq 60$ years, although only $30 \%$ of episodes were due to nosocomial meningitis. In our cohort, patients $\geq 65$ years had significantly higher mortality risk. No significant difference was found regarding compli- cations and non-alert state at discharge between the elderly and non-elderly patients.

Immunocompromised state, immunosuppressive therapy, diabetes, and alcohol abuse are known risk factors for infection. In ICU patients, major risk factors for nosocomial infections include diabetes mellitus and steroid use [21]. Frontera et al. [10] found a significant association between diabetes and nosocomial meningitis. In our cohort, $12.9 \%$ of patients were diabetic, which is consistent with previous reports of $11 \%$ [22]. Similarly to Srihawan et al. [19], we did not find an association between immunocompromised state and outcome.

The associations between neuroinvasive procedures, head trauma, or subarachnoid hemorrhage and nosocomial meningitis are well established. Several previous studies have 
analyzed cohorts of nosocomial meningitis after CNS surgical intervention [17,22-25]. In contrast, nosocomial meningitis as a complication of bacteremia is rare [4]. We estimated a $7.1 \%$ incidence of nosocomial meningitis due to bacteremia, which is higher than expected. Nevertheless, all remaining patients had CNS-related risk factors for nosocomial meningitis.

Fever and altered mental status were the most frequent clinical signs, consistent with current evidence [4]. However, these characteristics are nonspecific and often unhelpful for differential diagnosis of possible foci of infection, especially in critical patients. In a prospective study in a neurologic ICU, fever occurred in nearly $25 \%$ of patients, of which more than half (52\%) were noninfectious [26]. In severely brain-injured patients, this proportion is even higher, reaching $78 \%$ during the first week of hospitalization [27]. Altered mental status can have a multitude of possible causes, including drugs, sepsis, electrolyte disturbances, hypercarbia, and hypoxemia, none of which are rare in an ICU. Moreover, the predisposing condition for meningitis itself (head injury, intracranial bleeding, subarachnoid hemorrhage, or tumor) can cause decreased consciousness. Nonetheless, fever and/or deteriorating mental status in the absence of another clear source of infection and in the context of recent head trauma or neurosurgery should always raise suspicion for CNS infection and prompt CSF analysis.

Shi et al. [28] concluded that a GCS score $<9$ was related to substandard outcomes, and Erdem et al. [29] described GCS score $<10$ as an independent risk factor for mortality, while Kim et al. [22] did not find any association between a semicomatose or comatose mental state and mortality. We did not observe any association between GCS score at diagnosis and mortality.

Abnormalities in CSF cell count, glucose, and protein might be unreliable indicators for infection presence in patients with health-care associated ventriculitis and meningitis [11]. For instance, surgery, trauma, and intracranial hemorrhage can all induce CSF abnormalities, and aseptic inflammation can result from tissue response to injury or stimulation by noninfectious agents [13]. CSF pleocytosis, including polymorphonuclear pleocytosis, is particularly unreliable, as it can be reactive to prior neurosurgical interventions or devices [12]. Two previous studies have shown that a low CSF glucose level correlated with higher mortality [20,29], but two other studies did not show such an association [19,22]. In our cohort, we did not find an association between CSF glucose level and mortality.
It is estimated that at least $11 \%-30 \%$ of nosocomial meningitis is culture negative [1]. We observed an elevated rate of culture-negative CNS infection (70\%), which we postulate might be due to high selective pressure by antibiotics in ICU patients or over-valorization of clinical and analytical CSF features. According to CDC criteria, fever and CSF pleocytosis with elevated protein and/or decreased glucose and appropriate antimicrobial therapy are the criteria for diagnosis of nosocomial meningitis. Despite fulfilling these criteria, we suspect that meningeal reactions to TBI or cerebral hemorrhage, for example, are over-diagnosed as meningitis.

Cutaneous Gram-positive bacteria, such as coagulase-negative Staphylococci, Staphylococcus aureus, and Cutibacterium acnes, are the main etiological agents of nosocomial meningitis [1]. However, the proportion of Gram-negative infections is increasing [1,3], with a reported prevalence as high as $52 \%$ [2]. Accordingly, we observed a higher incidence of Gram-negative meningitis ( $15.7 \%$ vs. $8.6 \%$ ), but the number of culture-positive cases was too low to draw conclusions. When comparing meningitis due to Gram-negative bacteria to meningitis due to Gram-positive bacteria, some studies found that the former was associated with higher mortality $[2,18]$. In contrast, we did not observe a significant difference in mortality among culture-positive and culture-negative meningitis.

As expected, therapy with steroids was not associated with lower mortality. Although attenuation of subarachnoid space inflammatory response by steroid use might be effective in decreasing many of the pathophysiologic consequences of bacterial meningitis (cerebral edema, increased intracranial pressure, altered cerebral blood flow, cerebral vasculitis, and neuronal injury), these patients generally require antimicrobial therapy with vancomycin (at least during the empiric phase of antimicrobial therapy), and the diminished inflammatory response induced by dexamethasone might reduce CSF vancomycin penetration [30]. Steroid use was associated with a higher complication rate, possibly because steroids are mostly used in this setting.

The mortality rate of nosocomial meningitis ranges from 8\%-50\% [2,18-20,23,28,29,31,32]. Most studies are of patients with post-neurosurgical or post-traumatic meningitis and do not distinguish between critically and non-critically ill patients, which can be a confusing factor when comparing it to our cohort. Two studies reported mortality rates of $39.7 \%$ [2] and 50\% [33] in post-neurosurgical critically ill patients with nosocomial meningitis. The mortality rate of our cohort, $37.1 \%$, was similar to that of previous studies. 
This study has some limitations. First, it is a retrospective single-center study, with only 70 patients. Second, we included all patients that fulfilled CDC criteria for nosocomial meningitis, regardless of negative CSF cultures or absence of CSF analysis, which might have resulted in an over-estimation incidence. The low pathogen isolation rate also hindered analysis of etiological agents. Multi-center prospective studies with larger populations are needed to improve knowledge and practices on diagnosis, therapy, and outcomes of nosocomial meningitis.

In this study, nosocomial meningitis in critical care had a low incidence rate $(0.75 \%)$ but a high mortality rate $(37.1 \%)$ and a significant complication rate (47.1\%). Advanced age ( $\geq 65$ years old) and complications were associated with higher hospital mortality. No clinically relevant factor was predictive of complication development.

Fever and altered mental status were the most consistent clinical signs; however, in critical care, these can be explained by a multitude of other causes. Additionally, this population is frequently prescribed broad-spectrum antibiotics, altering CSF pleocytosis, biochemistry parameters, and cultures. Defining meningitis by the CDC criteria in this population might be encouraging over-diagnosis and over-treatment of this condition. Conversely, an appropriate balance between THE risk of not treating a true CNS infection and THE risk of unnecessary broad-spectrum antibiotic use might be difficult to achieve.

\section{CONFLICT OF INTEREST}

No potential conflict of interest relevant to this article was reported.

\section{ACKNOWLEDGMENTS}

The authors thank Dr. Irene Aragão for her valuable contribution to the concept and design of this project.

\section{ORCID}

Sofia R. Valdoleiros

Cristina Torrão

Laura S. Freitas

Diana Mano

Celina Gonçalves

Carla Teixeira https://orcid.org/0000-0003-4283-7549 https://orcid.org/0000-0003-1585-6664 https://orcid.org/0000-0001-6214-2221 https://orcid.org/0000-0003-4092-9941 https://orcid.org/0000-0002-0194-5140 https://orcid.org/0000-0002-9581-0756

\section{AUTHOR CONTRIBUTIONS}

Conceptualization: SRV, CT, LSF, CG, CT. Data curation: SRV, CT, LSF, DM. Formal analysis: SRV, CT, LSF, DM, CG. Methodology: SRV, CT, LSF, DM. Project administration: SRV, CT. Validation: SRV, CT, CG. Visualization: SRV, CT. Writing-original draft: SRV, CT, LSF, DM. Writing-review \& editing: SRV, CT, LSF, CG, CT.

\section{SUPPLEMENTARY MATERIALS}

Supplementary materials can be found via https://doi. org/10.4266/acc.2021.01151.

\section{REFERENCES}

1. Robinson CP, Busl KM. Meningitis and encephalitis management in the ICU. Curr Opin Crit Care 2019;25:423-29.

2. Kurtaran B, Kuscu F, Ulu A, Inal AS, Komur S, Kibar F, et al. The causes of postoperative meningitis: the comparison of Gram-negative and Gram-positive pathogens. Turk Neurosurg 2018;28:589-96.

3. Hussein K, Bitterman R, Shofty B, Paul M, Neuberger A. Management of post-neurosurgical meningitis: narrative review. Clin Microbiol Infect 2017;23:621-8.

4. van de Beek D, Drake JM, Tunkel AR. Nosocomial bacterial meningitis. N Engl J Med 2010;362:146-54.

5. Baer ET. Post-dural puncture bacterial meningitis. Anesthesiology 2006;105:381-93.

6. Baltas I, Tsoulfa S, Sakellariou P, Vogas V, Fylaktakis M, Kondodimou A. Posttraumatic meningitis: bacteriology, hydrocephalus, and outcome. Neurosurgery 1994;35:422-6.

7. Choi D, Spann R. Traumatic cerebrospinal fluid leakage: risk factors and the use of prophylactic antibiotics. Br J Neurosurg 1996;10:571-5.

8. Bullock MR, Chesnut R, Ghajar J, Gordon D, Hartl R, Newell DW, et al. Surgical management of depressed cranial fractures. Neurosurgery 2006;58(3 Suppl):S56-60.

9. Hoogmoed J, van de Beek D, Coert BA, Horn J, Vandertop WP, Verbaan D. Clinical and laboratory characteristics for the diagnosis of bacterial ventriculitis after aneurysmal subarachnoid hemorrhage. Neurocrit Care 2017;26:362-70.

10. Frontera JA, Fernandez A, Schmidt JM, Claassen J, Wartenberg KE, Badjatia N, et al. Impact of nosocomial infectious complications after subarachnoid hemorrhage. Neurosurgery 2008;62:80-7. 
11. Tunkel AR, Glaser CA, Bloch KC, Sejvar JJ, Marra CM, Roos KL, et al. The management of encephalitis: clinical practice guidelines by the Infectious Diseases Society of America. Clin Infect Dis 2008;47:303-27.

12. Busl KM. Nosocomial infections in the neurointensive care unit. Neurol Clin 2017;35:785-807.

13. Zarrouk V, Vassor I, Bert F, Bouccara D, Kalamarides M, Bendersky $\mathrm{N}$, et al. Evaluation of the management of postoperative aseptic meningitis. Clin Infect Dis 2007;44:1555-9.

14. Schade RP, Schinkel J, Roelandse FW, Geskus RB, Visser LG, van Dijk JM, et al. Lack of value of routine analysis of cerebrospinal fluid for prediction and diagnosis of external drainage-related bacterial meningitis. J Neurosurg 2006;104:101-8.

15. Horan TC, Andrus M, Dudeck MA. CDC/NHSN surveillance definition of health care-associated infection and criteria for specific types of infections in the acute care setting. Am J Infect Control 2008;36:309-32.

16. Governale LS, Fein N, Logsdon J, Black PM. Techniques and complications of external lumbar drainage for normal pressure hydrocephalus. Neurosurgery 2008;63(4 Suppl 2):379-84.

17. Palabiyikoglu I, Tekeli E, Cokca F, Akan O, Unal N, Erberktas I, et al. Nosocomial meningitis in a university hospital between 1993 and 2002. J Hosp Infect 2006;62:94-7.

18. Dizbay M, Tunçcan OG, Arman D, Baş S, Gürsoy A, Aktaş F. Factors associated with mortality in nosocomial central nervous system infections. ANKEM Derg 2011;25:6-11.

19. Srihawan C, Castelblanco RL, Salazar L, Wootton SH, Aguilera E, Ostrosky-Zeichner L, et al. Clinical characteristics and predictors of adverse outcome in adult and pediatric patients with healthcare-associated ventriculitis and meningitis. Open Forum Infect Dis 2016;3:ofw077.

20. Lu CH, Chang WN, Chuang YC, Chang HW. The prognostic factors of adult gram-negative bacillary meningitis. J Hosp Infect 1998;40:27-34.

21. Ozer B, Ozbakıs Akkurt BC, Duran N, Onlen Y, Savas L, Turhanoglu S. Evaluation of nosocomial infections and risk factors in critically ill patients. Med Sci Monit 2011;17:PH17-22.

22. Kim HI, Kim SW, Park GY, Kwon EG, Kim HH, Jeong JY, et al. The causes and treatment outcomes of 91 patients with adult nosocomial meningitis. Korean J Intern Med 2012;27:171-9.
23. Wang KW, Chang WN, Huang CR, Tsai NW, Tsui HW, Wang $\mathrm{HC}$, et al. Post-neurosurgical nosocomial bacterial meningitis in adults: microbiology, clinical features, and outcomes. J Clin Neurosci 2005;12:647-50.

24. Logigan C, Mihalache D, Dorneanu O, Turcu T. Study of nosocomial bacillary meningitis admitted in the Clinic of Infectious Diseases Iaşi on a 20 years period. Rev Med Chir Soc Med Nat Iasi 2009;113:721-6.

25. Dimitriu SM, Luca V, Turcu T, Scurtu C, Prisecaru L. Acute nosocomial bacterial meningitis: apropos 27 cases. Rev Med Chir Soc Med Nat Iasi 1992;96:19-25.

26. Commichau C, Scarmeas N, Mayer SA. Risk factors for fever in the neurologic intensive care unit. Neurology 2003;60:837-41.

27. Stocchetti N, Rossi S, Zanier ER, Colombo A, Beretta L, Citerio G. Pyrexia in head-injured patients admitted to intensive care. Intensive Care Med 2002;28:1555-62.

28. Shi YJ, Zheng GH, Qian LY, Qsman RA, Li GG, Zhang GJ. Longitudinal analysis of risk factors for clinical outcomes of enterobacteriaceae meningitis/encephalitis in post-neurosurgical patients: a comparative cohort study during 2014-2019. Infect Drug Resist 2020;13:2161-70.

29. Erdem I, Hakan T, Ceran N, Metin F, Akcay SS, Kucukercan M, et al. Clinical features, laboratory data, management and the risk factors that affect the mortality in patients with postoperative meningitis. Neurol India 2008;56:433-7.

30. Tunkel AR, Hartman BJ, Kaplan SL, Kaufman BA, Roos KL, Scheld WM, et al. Practice guidelines for the management of bacterial meningitis. Clin Infect Dis 2004;39:1267-84.

31. Federico G, Tumbarello M, Spanu T, Rosell R, Iacoangeli M, Scerrati M, et al. Risk factors and prognostic indicators of bacterial meningitis in a cohort of 3580 postneurosurgical patients. Scand J Infect Dis 2001;33:533-7.

32. Weisfelt M, van de Beek D, Spanjaard L, de Gans J. Nosocomial bacterial meningitis in adults: a prospective series of 50 cases. J Hosp Infect 2007;66:71-8.

33. Chouhdari A, Ebrahimzadeh K, Rezaei O, Samadian M, Sharifi G, Hajiesmaeili M. Investigating related factors with mortality rate in patients with postoperative meningitis: One longitudinal follow up study in Iran. Iran J Neurol 2018;17:82-5. 